\title{
ELLIPTIC, HYPERBOLIC, AND CONDENSER CAPACITY; GEOMETRIC ESTIMATES FOR ELLIPTIC CAPACITY
}

\author{
DIMITRIOS BETSAKOS
}

\begin{abstract}
The elliptic capacity of a compact set $E$ in the plane is defined by R.Kühnau by least energy considerations analogous to the definition of logarithmic capacity but using the elliptic distance in place of the euclidian distance. We prove an identity connecting elliptic capacity, extremal length, and the capacity of the condenser with plates $E$ and $E^{*}$, where $E^{*}$ is antipodal to $E$ in the Riemann sphere. An analogous identity for hyperbolic capacity, conjectured by P.Duren and J.Pfaltzgraff, is also established. We show that elliptic capacity is always smaller than hyperbolic capacity, thus answering a question of P.Duren and R.Kühnau. We prove some geometric estimates for elliptic capacity. These estimates involve geometric transformations such as polarization, circular symmetrization, projection, and shoving.
\end{abstract}

\section{INTRODUCTION}

This paper may be considered as a continuation of the paper [7] written by P.Duren and R.Kühnau. We study some properties of elliptic capacity and its relations with hyperbolic capacity, the capacity of condensers, and extremal length. We start with several definitions.

Let $E$ be a compact set in the extended complex plane $\widehat{\mathbb{C}}$ and let $\delta(z, \zeta)$ be a "distance function" on $\widehat{\mathbb{C}}$; (some interesting choices for $\delta$ will be specified below). If $\mu$ is a probability measure on $E$, then the energy of $\mu$ with respect to $\delta$ is defined by

$$
I_{\delta}(E)=\int_{E} \int_{E} \log \frac{1}{\delta(z, \zeta)} d \mu(z) d \mu(\zeta)
$$

(provided that the integral exists). The capacity of $E$ with respect to $\delta$ is defined by

$$
d_{\delta}(E)=\exp \left\{-\inf _{\mu} I_{\delta}(\mu)\right\}
$$

where the infimum is taken over all probability Borel measures on $E$. If there exists a measure $\mu$ on $E$ for which the above infimum is attained, then $\mu$ is the $\delta$-equilibrium measure of $E$. The corresponding potential

$$
u_{\delta}(z)=\int_{E} \log \frac{1}{\delta(z, \zeta)} d \mu(\zeta)
$$

Date: January 10, 2005.

1991 Mathematics Subject Classification. Primary: 31A15, 30C85.

Key words and phrases. Polarization, symmetrization, shove theorem, hyperbolic capacity, elliptic capacity, extremal length, condenser. 
is the $\delta$-conductor potential of $E$.

We now describe three specific choices for the distance function $\delta$. If $\delta(z, \zeta)=|z-\zeta|$, then the $\delta$-capacity of $E$ is the logarithmic capacity of $E$, denoted by $d(E)$.

If $E$ is a compact set in the unit disk $\mathbb{D}$ and

$$
\delta(z, \zeta)=\frac{|z-\zeta|}{|1-\bar{\zeta} z|}
$$

we obtain the hyperbolic capacity $d_{h}(E)$ of $E$.

If

$$
\delta(z, \zeta)=[z, \zeta]_{e}:=\frac{|z-\zeta|}{|1+\bar{\zeta} z|},
$$

then we obtain the elliptic capacity $d_{e}(E)$ of $E$. Elliptic capacity in this form was introduced by Kühnau [10]. Previously, M.Tsuji [15] had defined elliptic capacity in terms of the chordal distance, but Kühnau observed that his definition of elliptic capacity leads to developments that parallel closely the theory of hyperbolic capacity.

A concept closely related to the capacities defined above is the capacity of condensers. Suppose that $A, B$ are two nonempty, disjoint, compact sets, and that the set $\Omega:=\widehat{\mathbb{C}} \backslash(A \cup B)$ is connected. The triple $(\Omega, A, B)$ is a condenser. The sets $A, B$ are the plates and the domain $\Omega$ is the field of the condenser. If $\Omega \subset \mathbb{C}$, the capacity of $(\Omega, A, B)$ is defined by

$$
\operatorname{cap}(\Omega, A, B)=\operatorname{cap}(A, B)=\inf _{u} \mathcal{D}_{\Omega}(u),
$$

where

$$
\mathcal{D}_{\Omega}(u)=\int_{\Omega}|\nabla u|^{2} d m
$$

is the Dirichlet integral of $u, d m$ is the 2-dimensional Lebesgue measure, and the infimum is taken over all $C^{1}$ functions in $\Omega$ with boundary values 1 at $A$ and 0 at $B$; the capacity of an arbitrary condenser is defined by means of an auxiliary Möbius transformation. In Section 2 we review some facts about the capacity of condensers; we refer to [1], [3], [6], [11] for more information.

The antipodal point of the point $a \in \mathbb{C} \backslash\{0\}$ is the point $a^{*}=-1 / \bar{a}$. The points 0 and $\infty$ are also antipodal. Given a set $E \subset \widehat{\mathbb{C}}$, we define its antipodal set (or elliptic reflection) $E^{*}=\left\{a^{*}: a \in E\right\}$. Following [10], we call a set $E$ elliptically schlicht if $E \cap E^{*}=\emptyset$. At the other extreme $E$ is said to be diametrically symmetric if $E=E^{*}$. A closed elliptically schlicht set $E$ is said to be elliptically separated if there is a diametrically symmetric Jordan curve in $\widehat{\mathbb{C}}$ that separates $E$ from $E^{*}$. For an elliptically separated set $E$ let $\Omega$ be the largest diametrically symmetric domain in $\widehat{\mathbb{C}} \backslash\left(E \cup E^{*}\right)$ that contains the separating curve and let $C=E \cap \partial \Omega$. Duren and Kühnau [7, Theorem 1] showed that if $E$ is elliptically separated closed set and if $C$ consists of a finite number of smooth Jordan curves, then

$$
d_{e}(E)=\exp \left\{-\pi \lambda_{\Omega}\left(C, C^{*}\right)\right\}
$$


where $\lambda_{\Omega}\left(C, C^{*}\right)$ is the extremal distance between $C$ and $C^{*}$ with respect to $\Omega$. We refer to [14, Chapter II] for the definition and many properties of extremal distance.

We will prove the equality (1.1) in a more general setting which we now describe: Let $E$ be a closed set in $\widehat{\mathbb{C}}$ and suppose that $E$ and $E^{*}$ are separated by a Jordan curve $\gamma$. Let's assume that $E$ is contained in the interior $\operatorname{int}(\gamma)$ of $\gamma$ and $E^{*}$ is contained in the exterior $\operatorname{ext}(\gamma)$ of $\gamma$. Let $\Omega$ be the component of $\widehat{\mathbb{C}} \backslash\left(E \cup E^{*}\right)$ that contains $\gamma$. We define the sets

$$
\begin{aligned}
& A=(\widehat{\mathbb{C}} \backslash \Omega) \cap \operatorname{int}(\gamma), \\
& B=(\widehat{\mathbb{C}} \backslash \Omega) \cap \operatorname{ext}(\gamma) .
\end{aligned}
$$

We call the sets $A$ and $B$ the plates generated by $E$ and $E^{*}$ respectively. They are disjoint, compact sets in $\widehat{\mathbb{C}}$ and therefore $(\Omega, A, B)$ is a condenser. The set $C:=\partial A$ is the outer boundary of $E$.

Lemma 1. Under the above conditions, we have $C=\partial A=E \cap \partial \Omega, C^{*}=$ $\partial B, B=A^{*}, C \subset E \subset A, C^{*} \subset E^{*} \subset A^{*}$.

Theorem 1. Let $E$ be a compact set in $\widehat{\mathbb{C}}$. Assume that there exists a Jordan curve that separates $E$ from $E^{*}$. Let $\Omega$ be the component of $\widehat{\mathbb{C}} \backslash\left(E \cup E^{*}\right)$ that contains the separating curve and let $C$ be the outer boundary of $E$. Then

$$
d_{e}(E)=\exp \left\{-\pi \lambda_{\Omega}\left(C, C^{*}\right)\right\}=\exp \left\{-\pi / \operatorname{cap}\left(\Omega, A, A^{*}\right)\right\} .
$$

The connection of elliptic capacity with extremal length was proved in [7] via the Ahlfors-Beurling theorem which expresses extremal length in terms of the Dirichlet integral of a certain harmonic function; on the other hand, Dirichlet integrals are connected with energy integrals of measures through Green's formula. The requirement of smooth boundary in [7] comes (mainly) from this application of Green's formula. The extension to non-smooth boundaries in Theorem 1 is proved by a standard approximation argument.

Theorem 1 has another, more essential, element: the connection of elliptic capacity with condenser capacity. This is proved by least energy considerations and exploitation of the elliptic symmetry; extremal length is not used in this proof. The connection between elliptic and condenser capacity was implicit in [7] (at least for smooth boundaries) since both quantities are expressed in terms of Dirichlet integrals. Here we make this connection explicit; moreover, in the present article we make extensive use of condenser capacity as a tool (in addition to extremal length) for the study of elliptic capacity.

There exists a similar result for hyperbolic capacity. For a set $F \subset \widehat{\mathbb{C}}$, we let $F^{\#}=\{1 / \bar{z}: z \in F\}$ be its hyperbolic reflection.

Theorem 2. Let $E$ be a compact set in the unit disk $\mathbb{D}$ with outer boundary $C$. Let $\Omega$ be the component of $\mathbb{D} \backslash E$ which borders on the unit circle $\partial \mathbb{D}$. Then

$$
d_{h}(E)=\exp \left\{-2 \pi \lambda_{\Omega}(E, \partial \mathbb{D})\right\}=\exp \left\{-\pi / \operatorname{cap}\left(\Omega, A, A^{\#}\right)\right\}
$$


The first equality in (1.3) was proved by P.Duren and J.Pfaltzgraff [8, Theorem 1] under the additional assumption that $C$ consists of a finite number of smooth Jordan curves. Duren and Pfaltzgraff [8, pp. 208-209] conjectured that the first equality in (1.3) holds for all compact sets $E$. Theorem 2 verifies this conjecture. The comments we made above on the connection between elliptic and condenser capacity apply also here in the context of hyperbolic capacity. The proof of Theorem 2 is very similar to the proof of Theorem 1 and thus omitted.

In [7, p.323] it is also proved that for a closed, connected set $E \subset \mathbb{D}$ whose outer boundary $C$ is a smooth Jordan curve, we have $d_{e}(E) \leq d_{h}(E)$ with equality if and only if $C=-C$. Duren and Kühnau [7, p.323] ask whether this inequality holds for every closed set in $\mathbb{D}$. We answer this question in the affirmative:

Theorem 3. Let $E$ be a closed set in $\mathbb{D}$. Then

$$
d_{e}(E) \leq d_{h}(E)
$$

with equality if and only if $A \stackrel{\text { q.e. }}{=}-A$.

The expression $A \stackrel{\text { q.e. }}{=}-A$ means that the logarithmic capacity of the set $(A \backslash-A) \cup(-A \backslash A)$ is 0 . In general q.e. (quasi-everywhere) means "aside from a set of logarithmic capacity 0". In the proof of Theorem 3, there is an essential use of the connection of elliptic (hyperbolic) capacity with both extremal length and condenser capacity. The inequality (1.4) is proved by using only properties of extremal length. The proof of the equality statement makes use of the potential theory of condensers; in particular, the results of T.Bagby [3] play an essential role. In addition, an important tool is Brelot's generalized Dirichlet principle [5] with its equality statement.

The proofs of Lemma 1 and of Theorem 1 are in Section 2. The proof of Theorem 3 is in Section 3. In Section 4 we prove Theorem 4 (for the behavior of elliptic capacity under polarization) and Theorem 5 which is the analogous result for circular symmetrization and circular projection. The circular projection result asserts that the elliptic capacity of a compact set $E$ is larger than the elliptic capacity of the circular projection $E^{C}:=\{|z|: z \in E\}$ of $E$ onto the positive semi-axis; this result resembles the Beurling-Nevanlinna projection theorem for harmonic measure [13, Theorem 1, p.107]. Section 5 contains a shove theorem (Theorem 6 ) for elliptic capacity; according to this theorem, if $K$ is a closed subset of the interval [-1,1] and $K$ has total length $l$, then $d_{e}(K) \geq d_{e}\left(K^{S}\right)$, where $K^{S}=[-1,-1+l]\left(K^{S}\right.$ is formed by "shoving" the set $K$ towards the point -1$)$. Theorem 6 is analogous to the Beurling shove theorem for harmonic measure [13, p.110]. Theorems 5 and 6 lead to an explicit estimate for the elliptic capacity of a compact set involving the total length of the circular projection of the set on a ray emanating from the origin and complete elliptic integrals. This estimate is the content of Theorem 7 which is stated and proved in Section 6. Finally, Section 7 contains two open problems. 


\section{Proof of Theorem 1}

First we need to review some known results about condensers.

\section{Some facts about condensers}

The following facts summarize Theorems $1,2,3$ of [3]; see also [1]. Let $(\Omega, A, B)$ be a condenser such that $\infty \in \Omega$ and $\operatorname{cap}(\Omega, A, B)>0$. Let $\mathcal{S}(\Omega)$ be the family of all signed Borel measures of the form $\sigma=\sigma_{A}-\sigma_{B}$, where $\sigma_{A}$ is a probability measure on $A$ and $\sigma_{B}$ is a probability measure on $B$. The energy of $\sigma \in \mathcal{S}(\Omega)$ is

$$
I(\sigma)=\int_{A \cup B} \int_{A \cup B} \log \frac{1}{|z-\zeta|} d \sigma(z) d \sigma(\zeta) .
$$

There exists a unique $\tau \in \mathcal{S}(\Omega)$ (called the equilibrium measure of the condenser) such that $I(\tau)=2 \pi / \operatorname{cap}(\Omega, A, B)$. The function

$$
u(z)=\int_{A \cup B} \log \frac{1}{|z-\zeta|} d \tau(\zeta)
$$

is the equilibrium potential of the condenser. It is a function harmonic in $\Omega$ with constant boundary values $V_{A} \geq 0$ q.e. on $A$ and $V_{B} \leq 0$ q.e. on $B$. Moreover, $V_{A}-V_{B}=I(\tau)$.

Form now on, in this section $E$ will denote a compact set in $\widehat{\mathbb{C}}$ such that there exists a Jordan curve separating $E$ from its elliptic reflection $E^{*}$.

\section{Proof of Lemma 1}

First we prove that $C=E \cap \partial \Omega$. Let $\zeta \in C$, that is, $\zeta \in \partial A$. Then $\zeta \in \partial \Omega \cap \operatorname{int}(\gamma)$. We will prove that $\zeta \in E$. Suppose that $\zeta \notin E$. Then $\zeta$ belongs to a component $\Omega^{\prime}$ of $\widehat{\mathbb{C}} \backslash\left(E \cup E^{*}\right)$ with $\Omega^{\prime} \neq \Omega$. On the other hand $\zeta \in \partial \Omega$ and therefore $\zeta \in \bar{\Omega} \cap \overline{\Omega^{\prime}}$. However, the components of an open set have disjoint closures; contradiction.

Conversely, suppose $\zeta \in E \cap \partial \Omega$. Then $\zeta \in \operatorname{int}(\gamma)$. The boundary of $\Omega$ is decomposed into two sets $\partial \Omega=\partial A \cup \partial B$. Since $\partial A \subset \operatorname{int}(\gamma)$ and $\partial B \subset \operatorname{ext}(\gamma)$, we have $\zeta \in \partial A$.

From the equality $C=\partial A=E \cap \partial \Omega$, it follows at once that $C \subset E \subset A$.

Now we prove that $B=A^{*}$. Let $z \in B$. Then either $z \in E^{*}$ or $z \in B \backslash E^{*}$. If $z \in E^{*}$, then $z^{*} \in A$. Hence $z \in A^{*}$. If $z \in B \backslash E^{*}$, then $z \in \widehat{\mathbb{C}} \backslash \Omega$ and

$z \in \operatorname{ext}(\gamma)$. Therefore $z$ belongs to a component $\Omega_{1}$ of $\left(\widehat{\mathbb{C}} \backslash\left(E \cup E^{*}\right)\right) \cap \operatorname{ext}(\gamma)$ with $\Omega_{1} \neq \Omega$. Therefore, every curve that joins $z$ to $\gamma$ must intersect $E$. This implies that every curve that joins $z^{*}$ to $\gamma$ must intersect $E$ which means that $z^{*}$ belongs to a component $\Omega_{2}$ of $\left(\widehat{\mathbb{C}} \backslash\left(E \cup E^{*}\right)\right) \cap \operatorname{int}(\gamma)$ with $\Omega_{2} \neq \Omega$. Hence $z^{*} \in A$ and therefore $z \in A^{*}$. So we prove that $B \subset A^{*}$. The converse inclusion is proved in a similar way.

Finally, the equality $B=A^{*}$ implies that $C^{*} \subset E^{*} \subset A^{*}$ and $C^{*}=$ $\partial A^{*}$. 
From now on in this section we will denote by $A$ the plate generated by $E$, by $C$ the outer boundary of $E$, and by $\Omega$ the field of the condenser $\left(\Omega, A, A^{*}\right)$.

Lemma 2. Let $\tau=\tau_{A}-\tau_{A^{*}}$ be the equilibrium measure of the condenser $\left(\Omega, A, A^{*}\right)$. Then $\tau$ is diametrically symmetric in the sense that $\tau(F)=$ $-\tau\left(F^{*}\right)$ for every Borel set $F \subset A \cup A^{*}$.

Proof. By conformal invariance we may assume that $\infty \in \Omega$. We define a Borel signed measure $\hat{\tau}$ on $A \cup A^{*}$ as follows: if $F \subset A \cup A^{*}$, we set $\hat{\tau}(F)=-\tau\left(F^{*}\right)$. Then for the energy of $\hat{\tau}$ we have

$$
\begin{aligned}
I(\hat{\tau})= & \int_{C \cup C^{*}} \int_{C \cup C^{*}} \log \frac{1}{|z-\zeta|} d \hat{\tau}(z) d \hat{\tau}(\zeta) \\
= & \int_{C^{*}} \int_{C^{*}} \log \frac{1}{\left|z^{*}-\zeta^{*}\right|} d \tau_{A^{*}}(z) d \tau_{A^{*}}(\zeta) \\
& +\int_{C} \int_{C} \log \frac{1}{\left|z^{*}-\zeta^{*}\right|} d \tau_{A}(z) d \tau_{A}(\zeta) \\
& -\int_{C^{*}} \int_{C} \log \frac{1}{\left|z^{*}-\zeta^{*}\right|} d \tau_{A}(z) d \tau_{A^{*}}(\zeta) \\
& -\int_{C} \int_{C^{*}} \log \frac{1}{\left|z^{*}-\zeta^{*}\right|} d \tau_{A^{*}}(z) d \tau_{A}(\zeta) \\
= & I(\tau)+\int_{C^{*}} \int_{C^{*}} \log |z \zeta| d \tau_{A^{*}}(z) d \tau_{A^{*}}(\zeta)+\int_{C} \int_{C} \log |z \zeta| d \tau_{A}(z) d \tau_{A}(\zeta) \\
& -\int_{C^{*}} \int_{C} \log |z \zeta| d \tau_{A}(z) d \tau_{A^{*}}(\zeta)-\int_{C} \int_{C^{*}} \log |z \zeta| d \tau_{A^{*}}(z) d \tau_{A}(\zeta) \\
= & I(\tau)+2 \int_{C} \log |z| d \tau_{A^{*}}(z)+2 \int_{C} \log |z| d \tau_{A}(z)-\int_{C} \log |z| d \tau_{A}(z) \\
& -\int_{C^{*}} \log |\zeta| d \tau_{A^{*}}(\zeta)-\int_{C^{*}} \log |z| d \tau_{A^{*}}(z)-\int_{C} \log |\zeta| d \tau_{A}(\zeta) \\
= & I(\tau) .
\end{aligned}
$$

By the uniqueness of the equilibrium measure of condensers [1, Lemma 2], we conclude that $\tau=\hat{\tau}$ and therefore $\tau$ is diametrically symmetric.

Corollary 1. Let $\tau$ be the equilibrium measure of the condenser $\left(\Omega, A, A^{*}\right)$. Then

$$
I(\tau)=\min _{\sigma} I(\sigma)
$$

where the minimum is taken over all diametrically symmetric signed measures $\sigma \in \mathcal{S}(\Omega)$.

We shall prove now the equality

$$
d_{e}(E)=\exp \left\{-\pi / \operatorname{cap}\left(\Omega, A, A^{*}\right)\right\} .
$$




\section{Proof of (2.4)}

Since both $d_{e}(E)$ and $\operatorname{cap}\left(\Omega, A, A^{*}\right)$ are invariant under rotations of the Riemann sphere, we may assume that $\infty \in \Omega$. Let $\mu$ be the elliptic equilibrium measure of $E$. We define a Borel signed measure $\sigma$ on $E \cup E^{*}$ as follows: If $F$ is a Borel subset of $E$, we set $\sigma(F)=\mu(F)$. If $F$ is a Borel subset of $E^{*}$, we set $\sigma(F)=-\mu\left(F^{*}\right)$. Then $\sigma$ has total measure 0 , the positive part of $\sigma$ is the probability measure $\mu$ which is supported on $C$ and the negative part of $\sigma$ is a probability measure supported on $C^{*}$. The logarithmic energy of $\sigma[3, \mathrm{p} .318]$ is given by

$$
I(\sigma)=\int_{C \cup C^{*}} \int_{C \cup C^{*}} \log \frac{1}{|z-\zeta|} d \sigma(z) d \sigma(\zeta) .
$$

Using the construction of $\sigma$ above and a change of variables, we see that

$$
\begin{aligned}
I(\sigma)= & \int_{C} \int_{C} \log \frac{1}{|z-\zeta|} d \mu(z) d \mu(\zeta)+\int_{C} \int_{C} \log \frac{1}{\left|z^{*}-\zeta^{*}\right|} d \mu(z) d \mu(\zeta) \\
& -\int_{C} \int_{C} \log \frac{1}{\left|z-\zeta^{*}\right|} d \mu(\zeta) d \mu(z)-\int_{C} \int_{C} \log \frac{1}{\left|z^{*}-\zeta\right|} d \mu(\zeta) d \mu(z) \\
= & 2 \int_{C} \int_{C} \log \frac{|1+\bar{\zeta} z|}{|z-\zeta|} d \mu(z) d \mu(\zeta)+\int_{C} \int_{C} \log |z \zeta| d \mu(z) d \mu(\zeta) \\
& -\int_{C} \int_{C} \log |\zeta| d \mu(z) d \mu(\zeta)-\int_{C} \int_{C} \log |\zeta| d \mu(z) d \mu(\zeta) \\
= & 2 I_{e}(\mu) .
\end{aligned}
$$

From the computation above and Corollary 1 we conclude that $\sigma$ is the equilibrium measure of the condenser $\left(\Omega, A, A^{*}\right)$. Therefore the equality $I(\sigma)=2 I_{e}(\mu)$ is equivalent to $d_{e}(E)=\exp \left\{-\pi / \operatorname{cap}\left(\Omega, A, A^{*}\right)\right\}$.

From (2.4) and a well known property of condenser capacity [3, Lemma 1], [1, Lemma 3] we obtain the following result.

Lemma 3. Let $\left\{A_{n}\right\}$ be a decreasing sequence of compact sets in $\Omega$ with $\cap_{n=1}^{\infty} A_{n}=A$. Then

$$
\lim _{n \rightarrow \infty} d_{e}\left(A_{n}\right)=d_{e}(A) .
$$

To finish the proof of Theorem 1, it remains to prove the equality

$$
d_{e}(E)=\exp \left\{-\pi \lambda_{\Omega}\left(C, C^{*}\right)\right\} .
$$

\section{Proof of (2.6)}

Since both $d_{e}(E)$ and $\lambda_{\Omega}\left(C, C^{*}\right)$ are invariant under rotations of the sphere, we may assume that $\infty \notin \Omega$. Consider a sequence $\left\{A_{n}\right\}$ of compact sets such that $\partial A_{n}$ consists of a finite number of analytic Jordan curves, $A_{n+1}$ is contained in the interior of $A_{n}$, and $\cap_{n=1}^{\infty} A_{n}=A$; (it is well-known that such a sequence exists [9, pp. 318-319]). Then, because of Lemma 3 ,

$$
d_{e}(E)=d_{e}(A)=\lim _{n \rightarrow \infty} d_{e}\left(A_{n}\right)
$$


Also, by [14, Theorem 2.37],

$$
\lambda_{\Omega}\left(A, A^{*}\right)=\lim _{n \rightarrow \infty} \lambda_{\Omega_{n}}\left(A_{n}, A_{n}^{*}\right),
$$

where $\Omega_{n}=\widehat{\mathbb{C}} \backslash\left(A_{n} \cup A_{n}^{*}\right)$. By [7, Theorem 1],

$$
d_{e}\left(A_{n}\right)=\exp \left\{-\pi \lambda_{\Omega_{n}}\left(A_{n}, A_{n}^{*}\right)\right\}, \quad n=1,2, \ldots
$$

Now (2.6) follows from (2.7), (2.8), and (2.9).

\section{Proof of Theorem 3}

Suppose $E$ is a compact set in $\mathbb{D}$. Let $C$ be its outer boundary, $A$ be the plate generated by $E$, and $D$ be the component of $\mathbb{D} \backslash E$ which borders the unit circle, that is, $D=\mathbb{D} \backslash A$. We set $\Omega=\widehat{\mathbb{C}} \backslash\left(A \cup A^{*}\right)$ and $G=\widehat{\mathbb{C}} \backslash\left(A \cup A^{\#}\right)$. Then $\Omega=D \cup \partial \mathbb{D} \cup D^{*}$ and $G=D \cup \partial \mathbb{D} \cup D^{\#}$. By the symmetry of $G$ with respect to the unit circle, the symmetry properties of extremal length $[14$, Theorem 2.48, p.161], and the conformal invariance of extremal length [14, Theorem 2.11, p.80]

$$
\lambda_{G}\left(A, A^{\#}\right)=2 \lambda_{D}(A, \partial \mathbb{D})=\lambda_{D}(A, \partial \mathbb{D})+\lambda_{D}\left(A^{\#}, \partial \mathbb{D}\right) .
$$

Also, by Theorems 1 and 2 ,

$$
\begin{gathered}
d_{h}(E)=d_{h}(A)=\exp \left\{-2 \pi \lambda_{D}(A, \partial \mathbb{D})\right\}, \\
d_{e}(E)=d_{e}(A)=\exp \left\{-\pi \lambda_{\Omega}\left(A, A^{*}\right)\right\} .
\end{gathered}
$$

Therefore, to prove (1.4), it suffices to prove

$$
\lambda_{G}\left(A, A^{\#}\right) \leq \lambda_{\Omega}\left(A, A^{*}\right) .
$$

By a property of extremal length [14, Theorem 2.10, p.79],

$$
\lambda_{\Omega}\left(A, A^{*}\right) \geq \lambda_{D}(A, \partial \mathbb{D})+\lambda_{D^{*}}\left(A^{*}, \partial \mathbb{D}\right) .
$$

Since $A^{*}$ is a rotation of $A^{\#}$,

$$
\lambda_{D^{\#}}\left(\partial \mathbb{D}, A^{\#}\right)=\lambda_{D^{*}}\left(\partial \mathbb{D}, A^{*}\right)=\lambda_{D}(A, \partial \mathbb{D}) .
$$

Now (3.4) follows from (3.1), (3.5), and (3.6).

We proceed with the equality statement. If $d(E)=0$, then $d(A)=$ $d\left(A^{*}\right)=d\left(A^{\#}\right)=0$. It is easy to prove that we also have $d_{e}(E)=d_{h}(E)=0$ (see e.g. [1, Theorem 7]). Moreover, we trivially have $E \stackrel{\text { q.e. }}{=}-E$. From now on (in this proof), we assume that $d(E)>0$; this implies $d_{e}(E)>0$ and $d_{h}(E)>0$. We also note that $d_{e}(E)<1$, because $E$ is contained in a disk $K_{r}=\{|z| \leq r\}$ with $0<r<1$, and $d_{e}\left(K_{r}\right)=r$; see [7].

Suppose that $A \stackrel{\text { q.e. }}{=}-A$. Then $A \stackrel{\text { q.e. }}{=} A^{*}$. We set $A_{1}=A \cap(-A)$. Then $A$ can be written as a disjoint union $A=A_{1} \cup A_{2}$, where $d\left(A_{2}\right)=0$. Also $A^{\#}=A_{1}^{\#} \cup A_{2}^{\#}, A^{*}=A_{1}^{*} \cup A_{2}^{*}$ with $d\left(A_{2}^{\#}\right)=d\left(A_{2}^{*}\right)=0$, and $G \stackrel{\text { q.e. }}{=} \Omega$. By well-known properties of extremal length (see [14, Theorem 2.13 and Corollary p.78]),

$$
\lambda_{\Omega}\left(A, A^{*}\right)=\lambda_{\Omega}\left(A_{1}, A_{1}^{*}\right)
$$


and

$$
\lambda_{G}\left(A, A^{\#}\right)=\lambda_{\Omega}\left(A_{1}, A_{1}^{\#}\right) .
$$

Since $A_{1}=-A_{1}$, we have $A_{1}^{*}=A_{1}^{\#}$. Hence

$$
\lambda_{\Omega}\left(A_{1}, A_{1}^{*}\right)=\lambda_{G}\left(A_{1}, A_{1}^{\#}\right) .
$$

It follows from (3.7), (3.8), (3.9), and Theorems 1 and 2 that

$$
d_{e}(E)=d_{h}(E) \text {. }
$$

Conversely, suppose that $d_{e}(E)=d_{h}(E)$. Then

$$
\operatorname{cap}\left(\Omega, A, A^{*}\right)=\operatorname{cap}\left(G, A, A^{\#}\right) .
$$

By adding a constant (if necessary) to the equilibrium potential of the condenser $\left(\Omega, A, A^{*}\right)$, we obtain a function $u$, harmonic in $\Omega$ with boundary values $V$ q.e. on $A$ and $-V$ q.e. on $A^{*}$, where $V=-\log d_{e}(E)$. Similarly we obtain a function $v$, harmonic in $G$ with boundary values $V$ q.e. on $A$ and $-V$ q.e. on $A^{\#}$; (here we used our assumption $d_{e}(E)=d_{h}(E)$ ). Because of the symmetry of $G$ under hyperbolic reflection, we have $v=0$ on $\partial \mathbb{D}$ and $\mathcal{D}_{G}(v)=2 \mathcal{D}_{D}(v)$.

Consider the functions $u_{1}=\frac{u+V}{2 V}$ and $v_{1}=\frac{v+V}{2 V}$. By the generalized Dirichlet principle [5, Chapter II],

$$
\operatorname{cap}\left(\Omega, A, A^{*}\right)=\mathcal{D}_{\Omega}\left(u_{1}\right)=\frac{1}{4 V^{2}} \mathcal{D}_{\Omega}(u)
$$

and

$$
\operatorname{cap}\left(\Omega, A, A^{\#}\right)=\mathcal{D}_{G}\left(v_{1}\right)=\frac{1}{4 V^{2}} \mathcal{D}_{G}(v) .
$$

It follows from (3.11) that

$$
\mathcal{D}_{\Omega}(u)=\mathcal{D}_{G}(v) .
$$

We define a function $w$ in $\Omega$ as follows:

$$
w(z)= \begin{cases}v(z), & z \in D, \\ 0, & z \in \partial \mathbb{D}, \\ -v\left(z^{*}\right), & z \in D^{*} .\end{cases}
$$

Note that $w$ is continuous in $\Omega$, smooth in $\Omega \backslash \partial \mathbb{D}$, and has the boundary values $V$ q.e. on $A$ and $-V$ q.e. on $A^{*}$. Since the Dirichlet integral is invariant under reflection and rotation, $\mathcal{D}_{\Omega}(w)=2 \mathcal{D}_{D}(v)$. By the generalized Dirichlet principle [5, Chapter II],

$$
\mathcal{D}_{\Omega}(u) \leq \mathcal{D}_{\Omega}(w)=2 \mathcal{D}_{D}(v)=\mathcal{D}_{G}(v) .
$$

Because of (3.14) and the equality statement of the Dirichlet principle, the function $w$ is harmonic and equal to $u$ in $\Omega$. We conclude in particular that $u=0$ on $\partial \mathbb{D}$. By the reflection principle $u$ is extended to a function defined and harmonic in $S:=\widehat{\mathbb{C}} \backslash\left(A_{1} \cup A_{1}^{*}\right)$, where $A_{1}:=A \cap(-A)$.

Let $\zeta \in A \backslash A_{1}$. If $\zeta$ is an interior point of $A$, then $u$ is constant in a neighborhood of $\zeta$. Since $u=0$ on the unit circle, we infer that $u=0$ 
in $S$. Thus $V=0$ which means that $d_{e}(E)=1$; contradiction. Therefore $\zeta \in \partial A \backslash A_{1}$. Then $\zeta$ is an interior point of $S$. By the maximum principle, $u(\zeta)<V$. Hence $\zeta$ is an irregular (for the Dirichlet problem) point of the boundary of $\Omega$. By Kellogg's theorem [15, Theorem III.33.], the set of irregular points of a domain has logarithmic capacity 0 ; we conclude that $d\left(A \backslash A_{1}\right)=0$. This implies that $d\left(A^{*} \backslash A_{1}^{*}\right)=0$ and the proof of Theorem 3 is now complete.

\section{Elliptic CAPACITy, POlARIZATION, AND CIRCUlar SYMMETRIZATION}

We start with a brief review of polarization. For more information we refer to $[6, \S 2]$ and references therein. Let $\alpha$ be an oriented straight line in the plane. We denote by $-\alpha$ the straight line with opposite orientation. Let $\mathbb{C}_{\alpha}^{-}$and $\mathbb{C}_{\alpha}^{+}$be the closures of the half-planes into which $\alpha$ divides $\widehat{\mathbb{C}}$; the set $\mathbb{C}_{\alpha}^{-}$is chosen to be to the left of $\alpha$. Let $\widehat{F}$ denote the reflection in $\alpha$ of a set $F \subset \widehat{\mathbb{C}}$. We will also use the notation: $F^{-}=F \cap \mathbb{C}_{\alpha}^{-}, F^{+}=F \cap \mathbb{C}_{\alpha}^{+}$. The polarization of $F \subset \widehat{\mathbb{C}}$ with respect to $\alpha$ is the set

$$
\mathbf{P}_{\alpha}(F)=(F \cup \widehat{F})^{-} \cup(F \cap \widehat{F})^{+} .
$$

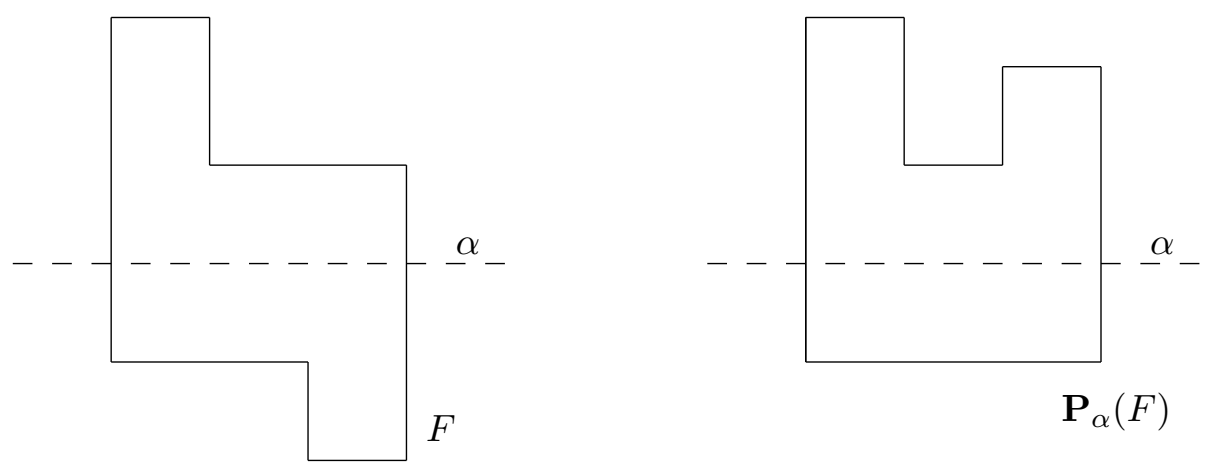

Figure 1: A set $F$ and its polarization $\mathbf{P}_{\alpha}(F)$.

If $(\Omega, A, B)$ is a condenser, then it is known $[6$, p.14] that

$$
\operatorname{cap}(\Omega, A, B) \geq \operatorname{cap}\left(\Omega^{\prime}, \mathbf{P}_{\alpha}(A), \mathbf{P}_{-\alpha}(B)\right),
$$

where $\Omega^{\prime}=\widehat{\mathbb{C}} \backslash\left(\mathbf{P}_{\alpha}(A) \cup \mathbf{P}_{-\alpha}(B)\right)$. It is also known [16] that if $E \subset \mathbb{C}$ is a compact set then $d(E) \geq d\left(\mathbf{P}_{\alpha}(E)\right)$. If, in addition, $E \subset \mathbb{D}$ and $\alpha$ passes through the origin, then $d_{h}(E) \geq d_{h}\left(\mathbf{P}_{\alpha}(E)\right)$. This inequality follows easily from (4.1) and Theorem 2. The same is true for elliptic capacity:

Theorem 4. Let $E$ be a compact set in $\widehat{\mathbb{C}}$ and let $\alpha$ be an oriented straight line that passes through the origin. Then

$$
d_{e}(E) \geq d_{e}\left(\mathbf{P}_{\alpha}(E)\right) .
$$


Proof. It is obvious that if $F \subset \mathbb{C}_{\alpha}^{+}$, then $F^{*} \subset \mathbb{C}_{\alpha}^{-}=\mathbb{C}_{-\alpha}^{+}$. This fact leads easily to the following equality

$$
\mathbf{P}_{-\alpha}\left(F^{*}\right)=\left(\mathbf{P}_{\alpha}(F)\right)^{*} .
$$

Now let $A$ be the plate generated by $E$. By Theorem 1, (4.1), and (4.3),

$$
\begin{aligned}
d_{e}(E) & =\operatorname{cap}\left(\Omega, A, A^{*}\right) \geq \operatorname{cap}\left(\Omega^{\prime}, \mathbf{P}_{\alpha}(A), \mathbf{P}_{-\alpha}\left(A^{*}\right)\right) \\
& =\operatorname{cap}\left(\Omega^{\prime}, \mathbf{P}_{\alpha}(A),\left(\mathbf{P}_{\alpha}(A)\right)^{*}\right)=d_{e}\left(\mathbf{P}_{\alpha}(A)\right) \geq d_{e}\left(\mathbf{P}_{\alpha}(E)\right) .
\end{aligned}
$$

The last inequality holds because $\mathbf{P}_{\alpha}(A) \supset \mathbf{P}_{\alpha}(E)$; (in fact, we may have strict inclusion).

By a standard method which involves a sequence of polarizations with respect to suitable oriented straight lines, we can use Theorem 4 to prove a result for the behavior of elliptic capacity under circular symmetrization. We refer to $[6, \S 3]$ and references therein for basic facts about circular symmetrization and the method mentioned above.

Theorem 5. Let $E$ be a compact set in $\widehat{\mathbb{C}}$ and let $E^{C}$ be the circular symmetrization of $E$ with respect to a ray emanating from the origin. Let $E^{P}$ be the circular projection of $E$ on this ray. Then

$$
d_{e}(E) \geq d_{e}\left(E^{C}\right) \geq d_{e}\left(E^{P}\right) .
$$

The last inequality follows from the inclusion $E^{P} \subset E^{C}$.

Remark: The assumption in Theorem 4 that $\alpha$ passes through the origin and the assumption that the ray in Theorem 5 emanates from the origin cannot be omitted. This can be seen by the consideration of linear segments and the use of the fact that elliptic capacity is not translation invariant.

\section{A SHOVE THEOREM FOR ELLIPTIC CAPACITY}

Our main tool in this section is the elliptic transfinite diameter [15], [10], [7]. Let $E$ be a compact set in the extended complex plane $\widehat{\mathbb{C}}$ with infinite number of points. The elliptic $n$-diameter of $E$ is defined by

$$
d_{e, n}(E)=\left\{\max _{z_{1}, z_{2}, \ldots, z_{n} \in E} \prod_{1 \leq j<k \leq n}\left[z_{j}, z_{k}\right]_{e}\right\}^{\frac{2}{n(n-1)}} .
$$

The sequence $\left\{d_{e, n}(E)\right\}_{n=1}^{\infty}$ is decreasing. The following is a remarkable characterization of elliptic capacity:

$$
d_{e}(E)=\lim _{n \rightarrow \infty} d_{e, n}(E) .
$$

The limit above is the elliptic transfinite diameter of $E$. For fixed $n \in \mathbb{N}, n$ points in $E$ for which

$$
d_{e, n}(E)=\left(\prod_{1 \leq j<k \leq n}\left[z_{j}, z_{k}\right]_{e}\right)^{\frac{2}{n(n-1)}}
$$


will be called elliptic $n$-Fekete points of $E$.

We will prove the following theorem which may be considered the analog for elliptic capacity of Beurling's shove theorem for harmonic measure.

Theorem 6. Let $K$ be a closed set in the interval $[-1,1]$ and let $K^{S}=$ $[-1,-1+l]$, where $l$ is the length of $K$. Then

$$
d_{e}(K) \geq d_{e}\left(K^{S}\right)
$$

Proof. By an approximation argument (based on Lemma 3), we may assume that $K$ consists of a finite number of closed intervals:

$$
K=\left[a_{1}, b_{1}\right] \cup\left[a_{2}, b_{2}\right] \cup \cdots \cup\left[a_{m}, b_{m}\right]
$$

with $-1 \leq a_{1}<b_{1}<a_{2}<b_{2}<\cdots<a_{m}<b_{m} \leq 1$. By applying a polarization with respect to the imaginary axis, we may also assume that

$$
-(K \cap[0,1]) \subset(K \cap[-1,0]) .
$$

If $b_{m}=1$, then $a_{1}=-1$. Therefore $d_{e}(K)=1$; in this case (5.1) is trivially true. So in the sequel we assume that $b_{m}<1$.

Lemma 4. If $K=\left[a_{1}, b_{1}\right]$, then $d_{e}(K) \geq d_{e}\left(K^{S}\right)$.

Proof. Because of (5.2), we have either $a_{1}<0<b_{1}, a_{1}<-b_{1}$, or $a_{1}<$ $b_{1}<0$. Let $\alpha$ be the vertical line with upward orientation which is such that $\mathbf{P}_{\alpha}\left\{b_{1}\right\}=\{-1\}$. By Theorem 1 and the polarization inequality for condensers (4.1),

$$
d_{e}(K)=\exp \left\{-\pi / \operatorname{cap}\left(K, K^{*}\right)\right\} \geq \exp \left\{-\pi / \operatorname{cap}\left(\mathbf{P}_{\alpha}(K), \mathbf{P}_{-\alpha}\left(K^{*}\right)\right)\right\} .
$$

It is easy to see that $\mathbf{P}_{\alpha}(K)=K^{S}$ and $\mathbf{P}_{-\alpha}\left(K^{*}\right)=K^{*}$. Hence

$$
\operatorname{cap}\left(\mathbf{P}_{\alpha}(K), \mathbf{P}_{-\alpha}\left(K^{*}\right)\right)=\operatorname{cap}\left(K^{S}, K^{*}\right) .
$$

The capacity of condensers is invariant under elliptic reflection. Therefore

$$
\operatorname{cap}\left(K^{S}, K^{*}\right)=\operatorname{cap}\left(\left(K^{S}\right)^{*}, K\right)=\operatorname{cap}\left(K,\left(K^{S}\right)^{*}\right) .
$$

Applying (4.1) again, we obtain

$$
\operatorname{cap}\left(K,\left(K^{S}\right)^{*}\right) \geq \operatorname{cap}\left(\mathbf{P}_{\alpha}(K), \mathbf{P}_{-\alpha}\left(\left(K^{S}\right)^{*}\right)\right)=\operatorname{cap}\left(K^{S},\left(K^{S}\right)^{*}\right) .
$$

Finally, by Theorem 1,

$$
d_{e}\left(K^{S}\right)=\exp \left\{-\pi / \operatorname{cap}\left(K^{S},\left(K^{S}\right)^{*}\right)\right\} .
$$

By (5.3) and (5.7) the lemma is proved.

Lemma 5. If $b_{m} \leq 0$, then $d_{e}(K) \geq d_{e}\left(K^{S}\right)$.

Proof. We choose $\alpha$ to be the vertical line with upward orientation and such that $\mathbf{P}_{\alpha}\left\{b_{m}\right\}=\left\{b_{m-1}\right\}$. Applying the method of Lemma 4 we prove that $d_{e}(K) \geq d_{e}\left(K_{1}\right)$, where $K_{1}$ consists of $m-1$ closed intervals. We continue inductively until we obtain one closed interval. Finally we apply Lemma 1.

Lemma 6. If $a_{m} \leq 0<b_{m}$, then $d_{e}(K) \geq d_{e}\left(K^{S}\right)$. 
Proof. The proof is similar to the proof of Lemmas 4 and 5. We now choose line $\alpha$ such that $\mathbf{P}\left\{b_{m}\right\}=\left\{b_{m-1}\right\}$ and we show that $d_{e}(K) \geq d_{e}\left(K_{1}\right)$, where $K_{1}$ consists of $m-1$ intervals:

$$
K_{1}=\left[a_{1}, b_{1}\right] \cup \cdots \cup\left[a_{m-1}, b_{m-1}^{\prime}\right] .
$$

If $b_{m-1}^{\prime}>0$, we repeat the same argument a finite number of times until either we obtain a single interval or the most right point of the set is nonpositive. In the first case we apply Lemma 4; in the second case we apply Lemma 5.

Lemma 7. Let $0 \leq a<b \leq 1$ and $\epsilon>0$. If either $a \geq 1 / 2, \epsilon \leq 1 / 4$ or $a<1 / 2, \epsilon \leq a$, then

$$
[a, b]_{e}[-b, b]_{e}[-b, a]_{e}>[a-\epsilon, b-\epsilon]_{e}[-b, b-\epsilon]_{e}[-b, a-\epsilon]_{e} .
$$

Proof. Recall that

$$
[z, \zeta]_{e}:=\frac{|z-\zeta|}{|1+\bar{\zeta} z|}
$$

Using this formula and doing the necessary calculations we see that (5.8) is equivalent to

$$
1+3 a b^{3}+2 b^{4}+2 b \epsilon+2 b^{2} \epsilon^{2}>a b+b^{2}+2 a b^{2} \epsilon+4 b^{3} \epsilon .
$$

To prove (5.9) it suffices to prove

$$
1+2 b^{4} \geq a b+b^{2}
$$

and

$$
3 a b^{3}+2 b \epsilon \geq 2 a b^{2} \epsilon+4 b^{3} \epsilon .
$$

It is easy to see that (5.10) is true for all $0 \leq a<b \leq 1$. It is also easy to see that (5.11) is true in both cases mentioned in the lemma.

Lemma 8. If $-1 \leq x_{1}<x_{2}<x_{3} \leq 1$, then $\left[x_{1}, x_{2}\right]_{e}<\left[x_{1}, x_{3}\right]_{e}$.

Proof. The inequality $\left[x_{1}, x_{2}\right]_{e}<\left[x_{1}, x_{3}\right]_{e}$ is equivalent to $x_{2}\left(1+x_{1}^{2}\right)<$ $x_{3}\left(1+x_{1}^{2}\right)$ which is true.

Suppose now that $a_{m}>0$. We define a number $\epsilon>0$ as follows:

- if $a_{m} \geq \frac{1}{2}$ and $a_{m}-b_{m-1} \geq \frac{1}{4}$, we set $\epsilon=\frac{1}{4}$;

- if $a_{m} \geq \frac{1}{2}$ and $a_{m}-b_{m-1}<\frac{1}{4}$, we set $\epsilon=a_{m}-b_{m-1}$;

- if $a_{m}<\frac{1}{2}$ and $b_{m-1} \leq 0$, we set $\epsilon=a_{m}$;

- if $a_{m}<\frac{1}{2}$ and $b_{m-1}>0$, we set $\epsilon=a_{m}-b_{m-1}$.

Let $K_{1}=\left(K \cap\left[-1, b_{m-1}\right]\right) \cup\left[a_{m}-\epsilon, b_{m}-\epsilon\right]$. We will prove that

$$
d_{e, n}\left(K_{1}\right) \leq d_{e, n}(K),
$$

for all sufficiently large $n \in \mathbb{N}$. We need first the following lemma.

Lemma 9. Every open interval $(x, y) \subset K_{1}$ contains elliptic $n$-Fekete points of $K_{1}$ for all sufficiently large $n \in \mathbb{N}$. 
Proof. Let $u$ be the elliptic conductor potential of $K_{1}$. It is a harmonic function in $\Omega=\widehat{\mathbb{C}} \backslash\left(K_{1} \cup K_{1}^{*}\right)$. Since the complement of $\Omega$ consists of a finite number of closed intervals, $\Omega$ is regular for the Dirichlet problem (see e.g. [11, p.299]). The harmonic function $u$ takes the boundary values $V$ on $K_{1}$ and $-V$ on $K_{1}^{*}$, where $V=-\log d_{e}\left(K_{1}\right)$. By a preliminary assumption we made at the beginning of this section, $d_{e}\left(K_{1}\right)<1$. Therefore the function $u$ is not constant, it is continuous everywhere (see [3, Theorem 2]) and attains its maximum value $V$ only on $K_{1}$. This implies that $u$ is superharmonic in a neighborhood of $K_{1}$. The Riesz measure $\mu$ of this superharmonic function coincides with the elliptic equilibrium measure of $K_{1}$; (see [4, Lemma 1] for a similar argument). On the other hand $\mu=-\Delta u$ in the sense of distributions $[11, \S 1.5]$.

If $\mu((x, y))=0$, then $u$ would be harmonic in a disk containing an interval $\left(x^{\prime}, y^{\prime}\right) \subset(x, y)$; because of the maximum principle this is absurd. So we have proved that the elliptic equilibrium measure of $(x, y)$ is positive.

By a well-known argument in potential theory [11, p.161], the measure $\mu$ is the vague limit of a subsequence of the sequence of discrete measures

$$
\mu_{n}=\frac{1}{n} \sum_{j=1}^{n} \delta_{z_{j}^{(n)}}
$$

where $\delta_{z_{j}^{(n)}}$ are unit point masses at the points $z_{j}^{(n)}$ which are elliptic $n$-Fekete points of $K_{1}$. It follows that the interval $(x, y)$ contains elliptic $n$-Fekete points of $K_{1}$ for all sufficiently large $n \in \mathbb{N}$.

Let $\tilde{z}_{1}, \tilde{z}_{2}, \ldots, \tilde{z}_{n} \in K_{1}$ be elliptic $n$-Fekete points of $K_{1}$, i.e.

$$
d_{e, n}\left(K_{1}\right)=\left(\prod_{1 \leq j<k \leq n}\left[\tilde{z}_{j}, \tilde{z}_{k}\right]_{e}\right)^{\frac{2}{n(n-1)}}
$$

Suppose that the first $l$ of these points belong to $K_{1} \cap\left[-1, b_{m-1}\right]$ and the rest belong to $\left[a_{m}-\epsilon, b_{m}-\epsilon\right]$, i.e. $\tilde{z}_{1}, \tilde{z}_{2}, \ldots, \tilde{z}_{l} \in K_{1} \cap\left[-1, b_{m-1}\right]$ and $\tilde{z}_{l+1}, \tilde{z}_{l+2}, \ldots, \tilde{z}_{n} \in\left[a_{m}-\epsilon, b_{m}-\epsilon\right]$. We consider the $n$ points $z_{1}, z_{2}, \ldots, z_{n}$ defined by

$$
z_{j}:=\tilde{z}_{j}, j=1,2, \ldots, l \text { and } z_{j}:=\tilde{z}_{j}+\epsilon, j=l+1, l+2, \ldots, n .
$$

These points belong to $K$. Therefore

$$
d_{e, n}(K) \geq\left(\prod_{1 \leq j<k \leq n}\left[z_{j}, z_{k}\right]_{e}\right)^{\frac{2}{n(n-1)}} .
$$

We will prove that

$$
\left(\prod_{1 \leq j<k \leq n}\left[z_{j}, z_{k}\right]_{e}\right)^{\frac{2}{n(n-1)}} \geq\left(\prod_{1 \leq j<k \leq n}\left[\tilde{z}_{j}, \tilde{z}_{k}\right]_{e}\right)^{\frac{2}{n(n-1)}} .
$$


Consider a pair of points $z_{j}, z_{k}$ with $l<j<k$. By Lemma 7,

$$
\left[z_{j}, z_{k}\right]_{e}\left[-z_{k}, z_{k}\right]_{e}\left[-z_{k}, z_{j}\right]_{e}>\left[\tilde{z}_{j}, \tilde{z}_{k}\right]_{e}\left[-z_{k}, \tilde{z}_{k}\right]_{e}\left[-z_{k}, \tilde{z}_{j}\right]_{e} .
$$

Now set

$$
f_{x}(y):=[x, y]_{e}[-y, y]_{e}[-y, x]_{e}, \quad x, y \in\left[a_{m}, b_{m}\right]
$$

and

$$
\tilde{f}_{x}(y):=[\tilde{x}, \tilde{y}]_{e}[-y, \tilde{y}]_{e}[-y, \tilde{x}]_{e}, \quad x, y \in\left[a_{m}, b_{m}\right] .
$$

Simple calculations show that the functions $f_{x}$ and $\tilde{f}_{x}$ have uniformly bounded derivatives with bounds depending only on sup $K$. Therefore, by an equicontinuity argument, the inequality (5.16) continues to hold if we replace $-z_{k}$ by a point in a neighborhood of $-z_{k}$. We choose an elliptic $n$-Fekete point $z_{k}^{\prime}$ in a neighborhood of $-z_{k}$; such a point exists by Lemma 9. So (5.16) holds with $-z_{k}$ replaced by $z_{k}^{\prime}$.

To prove (5.15) we decompose each of the products into two products $\Pi_{1}, \Pi_{2}$ and $\tilde{\Pi}_{1}, \tilde{\Pi}_{2}$ respectively. The product $\Pi_{1}$ contains triples of factors of the form

$$
\left[z_{j}, z_{k}\right]_{e}\left[z_{k}^{\prime}, z_{k}\right]_{e}\left[z_{k}^{\prime}, z_{j}\right]_{e}
$$

where $l<j<k$, and $z_{k}^{\prime}$ is an elliptic $n$-Fekete point in a neighborhood of $-z_{k}$ chosen as described above; the product $\Pi_{2}$ contains all the remaining factors of the first product. The products $\tilde{\Pi}_{1}, \tilde{\Pi}_{2}$ contain the corresponding factors of the other product in (5.15). It follows from Lemma 8 that $\Pi_{2} \geq \tilde{\Pi}_{2}$. Also each product of the form (5.17) in $\Pi_{1}$ is larger than the corresponding product in $\tilde{\Pi}_{1}$. Hence $\Pi_{1} \geq \tilde{\Pi}_{1}$ and $(5.15)$ is proved.

The inequality (5.12) follows now from (5.13), (5.14), and (5.15). Taking limits in (5.12) as $n \rightarrow \infty$ we obtain the inequality

$$
d_{e}(K) \geq d_{e}\left(K_{1}\right) .
$$

Let $\alpha$ be the imaginary axis and let $K_{2}=\mathbf{P}_{\alpha}\left(K_{1}\right)$. Then by Theorem 4 , $d_{e}\left(K_{1}\right) \geq d_{e}\left(K_{2}\right)$. Hence

$$
d_{e}(K) \geq d_{e}\left(K_{2}\right) .
$$

This inequality is the first step in an inductive argument. We continue by applying to $K_{2}$ the same procedure as we did for $K_{1}$ (that is, we shove $\epsilon$ units to the left the most right interval of $K_{2}$ ). After a finite number of such steps we arrive at a set

$$
K_{3}=\left[a_{1}^{\prime}, b_{1}^{\prime}\right] \cup \cdots \cup\left[a_{s}^{\prime}, b_{s}^{\prime}\right], \quad s \in \mathbb{N}
$$

such that one (at least) of the following cases occurs:

Case 1: $s=m-1$.

Case 2: $b_{s}^{\prime} \leq 0$.

Case 3: $a_{s}^{\prime}<0<b_{s}^{\prime}$.

In Case 1, Theorem 6 follows by induction on $m$. In Case 2, Theorem 6 follows by Lemma 5 . In Case 3, Theorem 6 follows by Lemma 6 . 


\section{A LOWER BOUND FOR ELLIPTIC CAPACITY}

We combine now Theorems 1, 5, and 6 to find an explicit lower bound for the elliptic capacity of a compact set in the unit disk.

Theorem 7. Let $K$ be a compact set in the unit disk $\mathbb{D}$ and let $l$ be the one-dimensional Lebesgue measure of the circular projection of $K$ on a ray emanating from the origin. Then

$$
d_{e}(K) \geq \exp \left\{-2 \pi \nu\left(\frac{l}{\sqrt{2\left(l^{2}-2 l+2\right)}}\right)\right\} .
$$

The function $\nu:(0,1) \rightarrow(0, \infty)$ is defined by

$$
\nu(r)=\frac{1}{4} \frac{\mathcal{K}\left(\sqrt{1-r^{2}}\right)}{\mathcal{K}(r)}, \quad \text { where } \quad \mathcal{K}(r)=\int_{0}^{1} \frac{d x}{\sqrt{\left(1-x^{2}\right)\left(1-r^{2} x^{2}\right)}}
$$

is the complete elliptic integral of the first kind; see e.g. $[14, \S 2.14,2.16],[2$, Ch. 3,5].

Proof. Let $K^{P}$ be the circular projection of $K$ on the radius $[-1,0]$ of $\mathbb{D}$. By Theorem 5,

$$
d_{e}(K) \geq d_{e}\left(K^{P}\right) .
$$

The length of $K^{P}$ is $l$. By Theorem 6 ,

$$
d_{e}\left(K^{P}\right) \geq d_{e}([-1,-1+l]) .
$$

By Theorem 1,

$$
d_{e}([-1,-1+l])=\exp \left\{-\pi \lambda\left([-1,-1+l],\left[1, \frac{1}{1-l}\right]\right)\right\} .
$$

By the translation invariance of extremal distance,

$$
\lambda\left([-1,-1+l],\left[1, \frac{1}{1-l}\right]\right)=\lambda\left([-l, 0],\left[2-l, 2-l+\frac{l}{1-l}\right]\right) .
$$

By [14, Theorem 2.74], the last extremal distance is equal to

$$
2 \nu\left(\frac{l}{\sqrt{2\left(l^{2}-2 l+2\right)}}\right) .
$$

The inequality (6.1) follows now from (6.2)-(6.6).

\section{OPEN PROBLEMS}

Problem 1. (Isoperimetric problem for elliptic capacity)

Among all compact sets in $\mathbb{D}$ with fixed area, which one has least elliptic capacity?

Problem 2. (A problem for logarithmic capacity)

Fix $r$ with $0<r<2$. Let $\mathcal{F}_{r}=\{K$ closed subset of $[-1,1]$ with length $r\}$. Find $\sup \left\{d(K): K \in \mathcal{F}_{r}\right\}$. 


\section{REFERENCES}

1. G.D.Anderson, M.K.Vamanamurthy, The Newtonian capacity of a space condenser, Indiana Univ. Math. J. 34 (1985), 753-776.

2. G.D.Anderson, M.K.Vamanamurthy, M.Vuorinen, Conformal Invariants, Inequalities, and Quasiconformal Maps. Wiley, 1997.

3. T. Bagby, The modulus of a plane condenser. J. Math. Mech. 17 (1967), 315-329.

4. D.Betsakos, On the equilibrium measure and the capacity of certain condensers. Illinois J. Math. 44 (2000), 681-689.

5. M.Brelot, Étude et extensions du principe de Dirichlet. Ann. Inst. Fourier 5 (1955), 371-419.

6. V.N.Dubinin, Symmetrization in the geometric theory of functions of a complex variable. (Russian) Uspekhi Mat. Nauk 49 (1994), 3-76; translation in Russian Math. Surveys 49 (1994), 1-79.

7. P.Duren, R.Kühnau, Elliptic capacity and its distortion under conformal mapping. J. Analyse Math. 89 (2003), 317-335.

8. P. Duren, J. Pfaltzgraff, Hyperbolic capacity and its distortion under conformal mapping. J. Analyse Math. 78 (1999), 205-218.

9. O.D. Kellogg, Foundations of Potential Theory. Dover Publications, 1953.

10. R. Kühnau, Transfiniter Durchmesser, Kapazität und Tschebyschewsche Konstante in der euklidischen, hyperbolischen und elliptischen Geometrie. J. Reine Angew. Math. 234 (1969), 216-220.

11. N.S.Landkof, Foundations of Modern Potential Theory. Springer-Verlag, 1972.

12. F. Leja, Points extremaux des ensembles et leur application dans la théorie des fonctions. Ann. Acad. Sci. Fenn. Ser. A. I, no. 250/20 (1958) 1-6.

13. R.Nevanlinna, Analytic Functions. Springer, New York 1970 (first edition in German 1936).

14. M. Ohtsuka, Dirichlet Problem, Extremal Length and Prime Ends. Van Nostrand Reinhold 1970.

15. M. Tsuji, Potential Theory in Modern Function Theory. Maruzen, 1959.

16. V.Wolontis, Properties of conformal invariants. Amer. J. of Math. 74 (1952), 587-606.

Department of Mathematics, Aristotle University of Thessaloniki, 54124

Thessaloniki, Greece

E-mail address: betsakos@math.auth.gr 